\title{
Comportamento sedentário em adolescentes brasileiros: uma revisão sistemática
}

\section{Sedentary behavior in Brazilian adolescents: a systematic review}

\section{AUTORES \\ Rodolfo Carlos dos Santos Silva Filho ${ }^{1}$ (D) Thiago Matheus Mendes Alves e Lemes ${ }^{1}$ (D) Jeffer Eidi Sasaki ${ }^{1}$ (D) \\ Alex Pinheiro Gordia ${ }^{2}$ (D) \\ Alynne Christian Ribeiro Andaki \\ 1 Universidade Federal do Triângulo Mineiro Programa de Pós-Graduação em Educação Física, Uberaba, Minas Gerais, Brasil. \\ 2 Universidade Federal do Recôncavo da Bahia, Centro de Formação de Professores, Amargosa, Bahia, Brasil.}

\section{CONTATO}

Rodolfo Carlos dos Santos Silva Filho rodolfo9405@hotmail.com

Rua Payaguas, n. 233, Ituverava,

São Paulo, Brasil.

CEP: $14500-000$.

DOI

$10.12820 /$ rbafs. $25 \mathrm{e} 0139$

\section{(cc) BY}

Este trabalho está licenciado com uma Licença Creative Commons - Atribuição 4.0 Internacional.

\begin{abstract}
RESUMO
Objetivou-se descrever aspectos metodológicos da pesquisa em comportamento sedentário e identificar intervenções para redução do comportamento sedentário em adolescentes brasileiros. A busca de dados foi de outubro de 2014 a abril de 2019. As bases de dados utilizadas foram LILACS, Pubmed, SciELO e Scopus. Foram critérios de inclusão artigos originais publicados em revistas nacionais e internacionais, teses e dissertações completas disponíveis para download, com amostras que incluíssem adolescentes brasileiros. Encontrou-se 491 estudos e 38 foram incluídos na síntese qualitativa. Somente $13,1 \%$ dos trabalhos apresentaram desenho experimental. O tempo de tela foi o indicador mais utilizado para caracterizar o comportamento sedentário. $O$ ponto de corte mais frequente foi o de 2 horas/dia, entretanto, identificou-se 17 pontos de corte diferentes. Apenas 13,1\% das pesquisas utilizaram medidas objetivas para mensurar o comportamento sedentário. Conclui-se que a falta de padronização nos indicadores e pontos de corte do comportamento sedentário dificultam a comparação entre os resultados das pesquisas. É necessária a utilização de medidas objetivas para informações mais fidedignas. Há carência de estudos de intervenção na redução do comportamento sedentário em adolescentes brasileiros.
\end{abstract}

Palavras-chave: Adolescente; Comportamento sedentário; Tempo de tela.

\section{ABSTRACT}

The aim of this study was to describe methodological aspects of the research on sedentary behavior and to identify interventions to reduce sedentary behavior in Brazilian adolescents. Data search was from October 2014 to April 2019. The databases used were LILACS, Pubmed, SciELO and Scopus. Inclusion criteria were original articles published in national and international journals, complete thesis and master thesis available for download, with samples that included Brazilian adolescents. 491 studies were found and 38 were included in the qualitative synthesis. Only $13.1 \%$ presented experimental design. Screen time was the most used indicator to characterize the sedentary behavior. The most frequent cutoff point was 2 hours/day; however, 17 different cutoff points were identified. Only 13.1\% of the surveys used objective measures to identify the sedentary behavior. It is concluded that the lack of standardization in the indicators and cutoff points of sedentary behavior make it difficult to compare the results of the research. It is necessary to use objective measures for more reliable information. There is a lack of intervention studies in the reduction of sedentary behavior of Brazilian adolescents.

Keywords: Adolescent; Sedentary behavior; Screen time.

\section{Introdução}

A adolescência é marcada por expressivas alterações de ordem social, fisiológica e comportamental, o que torna importante o estudo do comportamento sedentário nessa fase da vida ${ }^{1}$. Ainda, é sabido que a elevada exposição ao comportamento sedentário provoca efeitos deletérios à saúde do adolescente (independentemente do nível de atividade física), como alterações nos hábitos alimentares, obesidade, diabetes e síndrome metabólica ${ }^{2,3}$.

O elevado tempo de imobilização do músculo esquelético estimula a produção de lipídeos no fígado, acumula adipócitos na região abdominal, diminui a aptidão cardiorrespiratória, aumenta a resistência à insu- lina e hiperglicemia ${ }^{4,5}$. Consequentemente, estimula o surgimento de doenças crônicas. Praticar bullying, sofrer bullying, faltar à escola e apresentar desordens psicológicas também podem estar relacionados ao elevado tempo sentado de adolescentes ${ }^{6}$. Ainda, verificou-se que $56,1 \%$ dos estudantes do $9^{\circ}$ ano do Ensino Fundamental relataram realizar atividades sentados por mais de três horas diárias, além de elevada prevalência de tempo de TV superior a mais de duas horas por $\mathrm{dia}^{7}$.

Houve crescente aumento de estudos sobre comportamento sedentário, contudo ainda há confusão em relação à sua terminologia. Como exemplo, podemos citar o uso inequívoco da palavra "sedentário" como si- 
nônimo de "fisicamente inativo", o que provoca confusão no público em geral e debates até mesmo entre especialistas ${ }^{8,9}$. Vale citar também a utilização de variados métodos e instrumentos de avaliação na mensuração do comportamento sedentário, demonstrando que não há uma "regra" a ser seguida quando se objetiva estimá-lo.

O comportamento sedentário pode ser definido como qualquer tipo de comportamento caracterizado por um gasto energético $\leq 1,5$ equivalente metabólico $(\mathrm{MET})^{10}$. Tem sido mensurado em pesquisas com adolescentes por meio da exposição ao tempo de tela - que engloba as medidas agrupadas ou individuais do tempo em frente à TV, computador, videogame, aparelhos celulares e tablets $^{11}$ - temática essa que necessita ser abordada e investigada nesta população.

Alterar o comportamento sedentário de crianças e adolescentes, por meio da redução do tempo despendido com TV, computador, videogame e demais equipamentos eletrônicos, pode ser um meio de promoção de saúde e prevenção da obesidade e de doenças crônicas futuras ${ }^{12}$. A identificação de estudos que busquem proporcionar a mudança desse hábito é de grande valia, tendo em vista a baixíssima prevalência de intervenções focadas especificamente no comportamento sedentário e o pequeno efeito oriundo das mesmas ${ }^{13,14}$. Neste sentido, o presente estudo teve como objetivos descrever aspectos metodológicos da pesquisa em comportamento sedentário, bem como identificar intervenções para redução do comportamento sedentário, baseado em estudos que incluíssem adolescentes brasileiros.

\section{Métodos}

A revisão sistemática seguiu a recomendação da metodologia Preferred Reporting Items for Systematic Reviews and Meta-Analyses (PRISMA) ${ }^{15}$. O protocolo do estudo foi registrado na base de dados do International Prospective Register of Systematic Reviews (PROSPE$\mathrm{RO}$ ). Para seleção dos estudos pertinentes aos objetivos da pesquisa, foram critérios de inclusão artigos originais publicados em revistas nacionais e internacionais, teses e dissertações completas disponíveis para download, com amostras que incluíssem adolescentes brasileiros. A faixa etária definida como "adolescência" foi de 10 a 19 anos de idade ${ }^{16}$. O critério de exclusão dos estudos foram revisões sistemáticas e meta-análises. A busca de dados foi limitada ao período de outubro de 2014 a abril de 2019.

As bases de dados utilizadas nas buscas foram $L I$ LACS, Pubmed, SciELO e Scopus. Os seguintes descri- tores foram utilizados, estilo de vida sedentário; tempo sedentário; adolescente; criança; exercício; projetos de pesquisa; ensaio clínico controlado aleatório; ensaio clínico; tempo de tela; tempo de tv; tempo sentado; postura reclinada; postura deitada; postura em pé, e seus respectivos em inglês.

As estratégias de busca utilizadas variaram pela especificidade de cada uma das bases selecionadas. No PubMed, por exemplo, os termos de pesquisa foram ("sedentary lifestyle"[Title/Abstract] AND child[Title/ Abstract] OR adolescent[Title/Abstract]; "sedentary lifestyle"[Title/Abstract] AND exercise[Title/Abstract] AND child[Title/Abstract] OR adolescent $\left[\mathrm{Ti}^{-}\right.$ tle/Abstract]; "research design"[Title/Abstract] AND "sedentary lifestyle"[Title/Abstract] AND child[Title/ Abstract] OR adolescent[Title/Abstract]; "randomized controlled trial"[Title/Abstract] AND "sedentary lifestyle"[Title/Abstract] AND child[Title/Abstract] OR adolescent[Title/Abstract]; "screen time"[Title/ Abstract] OR "TV time"[Title/Abstract] OR "sitting time"[Title/Abstract] OR "reclining posture" [Title/Abstract] OR "lying posture"[Title/Abstract] OR "standing posture"[Title/Abstract] OR "sedentary time"[Ttitle/Abstract] AND child[Title/Abstract] OR adolescent [Title/Abstract]; "clinical trial"[Title/Abstract] AND "sedentary lifestyle"[Title/Abstract] OR exercise[Title/Abstract] AND child[Title/Abstract] OR adolescent [Title/Abstract].

Nas bases de dados LILACS e SciELO, utilizou-se o operador booleano $O R$ para combinar os descritores com seus respectivos em inglês e fez-se uso de truncagem com as palavras brasil \$ e brasileiro \$. Para as bases Pubmed e Scopus, utilizou-se apenas os termos em inglês e truncagem com brazil \$ e brazilian \$. Todos os descritores foram extraídos dos "Descritores em Ciências da Saúde" (DeCS). Os filtros utilizados para as bases de dados foram texto completo disponível; idioma inglês e/ou português; e anos de publicação 2014 a 2019. Foram utilizados nas bases os seguintes índices: LILACS (título, resumo, assunto); Pubmed (titlel abstract); SciELO (todos os índices); Scopus (article title, abstract, keywords).

Após a realização da busca de dados, o processo de exclusão deu-se primeiramente com os trabalhos que estavam em duplicata. Posteriormente, seguiu-se a ordem para a exclusão por títulos, resumos e leitura na íntegra dos trabalhos restantes. A seleção dos trabalhos foi realizada por pares de pesquisadores, individualmente e de forma cega. Assim, não houve divergência 
na seleção de trabalhos entre os pesquisadores.

Dos estudos incluídos na síntese qualitativa, foram extraídos os dados referentes à autoria de cada publicação, ano de publicação, ano e local de coleta, número amostral, faixa etária ou ano escolar, instrumento utilizado na mensuração do comportamento sedentário, modo de seleção da amostra, classificação do comportamento sedentário quanto ao tipo de variável (desfecho ou exposição), desenho de pesquisa, variáveis possivelmente associadas ao comportamento sedentário (ex.: nutrição, atividade física, obesidade, características sociodemográficas), pontos de corte, indicadores do comportamento sedentário (medidas utilizadas para caracterizá-lo, ex.: tempo de tela, tempo de TV, tempo sentado) e prevalência do comportamento sedentário.

\section{Resultados}

Foram identificados inicialmente um total de 491 estudos nas bases de dados. Destes, 352 estavam em duplicata, o que resultou em 139 trabalhos rastreados. Após a leitura dos títulos e dos resumos, 58 trabalhos foram considerados elegíveis e lidos na íntegra. Houve exclusão de 20 estudos (pesquisas que não analisaram o comportamento sedentário $n=9$; amostra fora $\mathrm{da}$ faixa etária do estudo $\mathrm{n}=3$; definição de "sedentarismo" como ausência de atividade física $n=1$; definição

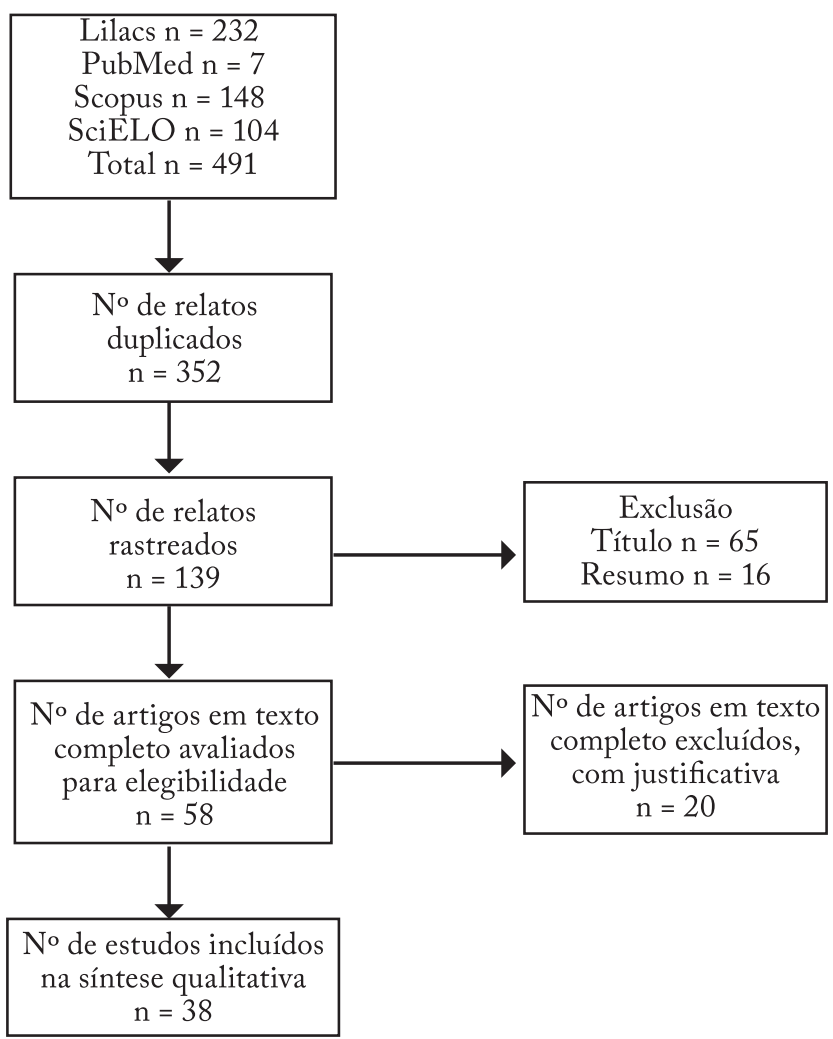

Figura 1 - Processo de seleção dos estudos avaliados. de "estilo de vida sedentário" como prática insuficiente de atividade física de moderada a vigorosa intensidade semanal < 300 minutos $n=2$; "estilo de vida sedentário" como prática de atividade física leve $\mathrm{n}=1$; publicações anteriores a outubro de $2014 \mathrm{n}=4$ ). Desse modo, 38 artigos (7,7\% do total) foram incluídos na síntese qualitativa (Figura 1).

Dos 38 trabalhos avaliados, os anos que tiveram mais publicações foram $2015(\mathrm{n}=10)$ e $2018(\mathrm{n}=10)$ - Figura 2.a. Sete estudos eram de representatividade nacional, dos quais seis utilizaram dados da Pesquisa Nacional de Saúde do Escolar (PeNSE, 2012 e 2015). A Figura 2.b mostra que, ao se excluir os trabalhos de representatividade nacional, a região que teve o maior número de coletas foi o $\mathrm{Sul}(\mathrm{n}=13)$. A região Centro-oeste teve um artigo $(n=1)$ e o Norte não foi local de coletas no pe-
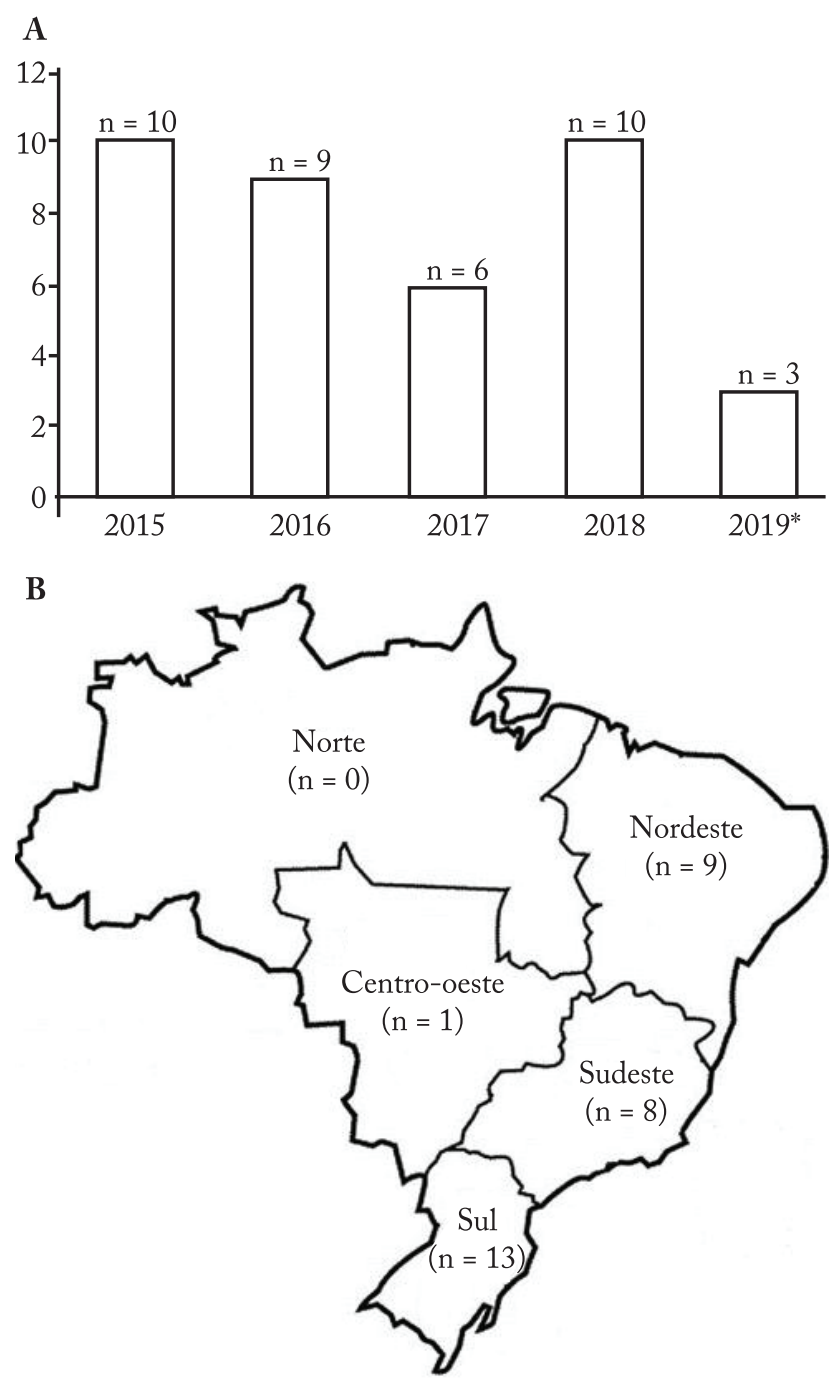

Figura 2 - (A) Distribuição do número de estudos por ano de publicação. (B) Distribuição do número de estudos por regiões brasileiras. Nota: data de busca foi limitada ao período de outubro de 2014 a abril de 2019. 
ríodo avaliado. O biênio 2011/2012 foi o período mais frequente em relação ao ano das coletas. As amostras variaram de $n=117$ a $n=109.104$ participantes. Quanto ao método de seleção, 26 estudos $(68,4 \%)$ definiram sua amostra de forma aleatória; os demais trabalhos o fizeram de forma não probabilística (Tabela 1 ).

Em relação às características metodológicas, foram encontrados cinco diferentes indicadores para mensuração do comportamento sedentário (tempo de tela, tempo de TV, tempo sentado, lazer sedentário, atividades sedentárias). O tempo de tela foi o indicador mais utilizado, seja isolado $\mathrm{n}=18$, somado ao tempo sentado $\mathrm{n}=8$, ou somado ao tempo de TV e tempo sentado $\mathrm{n}=$ 1 , totalizando $71 \%$ dos artigos $(n=27)$. Na identificação dos pontos de corte, para cada estudo que utilizou uma estratificação de variação em horas (ex.: $<2 \mathrm{~h} / \mathrm{d}$, 2-3 h/d, 3-4 h/d, > 4 h/d; < $1 \mathrm{~h} / \mathrm{d}$ até $>8 \mathrm{~h} / \mathrm{d} ;<1 \mathrm{~h} / \mathrm{d}$, $1 \mathrm{~h} / \mathrm{d}, 2 \mathrm{~h} / \mathrm{d}, 3 \mathrm{~h} / \mathrm{d}, 4 \mathrm{~h} / \mathrm{d}, \geq 5 \mathrm{~h} / \mathrm{d}$ ) contabilizou-se $\mathrm{n}=$ 1 ponto de corte. Assim, foram identificados 17 pontos de corte diferentes. Mínimo de duas horas por dia foi o ponto de corte mais adotado para determinar a exposição ao comportamento sedentário $(n=15 ; 39,4 \%)$, seja isolado $n=12$ ou somado a outro ponto de corte $n=3$. Cinco pesquisas $(13,1 \%)$ utilizaram medidas objetivas como meio de mensurar o comportamento sedentário. Os demais estudos utilizaram questionários (nacionais ou internacionais), ou derivaram seus dados de pesquisas nacionais ou internacionais (Tabela 2).

A Tabela 3 apresentou que, dos 38 artigos analisados, apenas $\mathrm{n}=5(13,1 \%)$ possuíram desenho de pesquisa experimental. A maioria dos trabalhos foi de corte transversal $(78,9 \%)$. Das variáveis que os estudos buscaram verificar associação com o comportamento sedentário, as mais frequentes foram aquelas relacionadas ao peso corporal (excesso de peso corporal, sobrepeso, obesidade; $n=9 ; 23,6 \%)$.

\section{Discussão}

Esta revisão objetivou descrever aspectos metodológicos da pesquisa em comportamento sedentário, além de identificar intervenções para redução do comportamento sedentário, baseado em estudos que incluíssem adolescentes brasileiros. Nossos resultados mostram a carência de intervenções dirigidas à redução da exposição ao comportamento sedentário. Somado a isso, nota-se a falta de consenso existente na literatura no que se refere ao modo de classificá-lo e mensurá-lo. Identificou-se grande variedade de pontos de corte e instrumentos entre os estudos. Estes achados também foram observados em outras revisões ${ }^{55,56}$.

A ausência de padronização traz resultados diferentes de prevalência do comportamento sedentário para uma mesma população. Estudo com adolescentes catarinenses identificou prevalência de comportamento sedentário maior que $70 \%$, com ponto de corte $\geq 2$ horas diárias ${ }^{57}$. Uma outra análise, com a mesma amostra, mostrou prevalência igual a $38,4 \%$, com o ponto de corte $\geq 4$ horas por dia ${ }^{58}$. A prevalência de exposição ao comportamento sedentário também apresentou grande variação entre as pesquisas incluídas nesta revisão, o que pode ser reflexo, justamente, da adoção de diferentes pontos de corte e instrumentos por parte dos pesquisadores da área. Ainda assim, conhecer o tempo que é despendido em atividades sedentárias é de suma importância para a elaboração de políticas públicas focadas na redução deste comportamento de risco ${ }^{59}$.

Outra questão que ainda carece de consenso entre pesquisadores diz respeito a semântica do termo "comportamento sedentário". Quatro estudos deixaram de ser incluídos em nossa síntese qualitativa devido a se referirem ao comportamento sedentário como ausência ou prática insuficiente de atividade física, ou ainda como prática de atividade física leve. Seria o comportamento sedentário apenas "inatividade física" com outro nome? Para buscar essa resposta já foram discutidos, entre pesquisadores, argumentos favoráveis e contrários ao questionamento ${ }^{8}$. Há quem defenda que comportamento sedentário e nível de atividade física não são variáveis opostas, mas sim independentes ${ }^{60}$. Porém, ainda é recorrente ambos os desfechos em saúde serem tratados de forma dependente e equívoca, como mostram os resultados do presente estudo. Por entendermos tratar-se de conceitos diferentes, ou seja, variáveis que possuem características e determinantes próprios e que assim se diferem quanto aos desfechos em saúde, sugerimos abordá-los de maneira independente.

Independentemente de qualquer tipo de debate, as pesquisas analisadas que investigaram a associação do comportamento sedentário a desfechos negativos à saúde corroboram as evidências encontradas na literatura. Ou seja, mostram que a elevada exposição ao comportamento sedentário se apresenta como importante fator de risco à vida dos adolescentes ${ }^{61-63}$. Aqueles que gastam mais tempo com TV e computador, por exemplo, possuem maiores chances de apresentar excesso de peso ${ }^{26}$. Adolescentes com maior exposição ao tempo de tela têm maior probabilidade de serem inativos fisicamente, consumirem menores quantidades de hortaliças e 
Tabela 1 - Características das amostras dos estudos avaliados.

\begin{tabular}{|c|c|c|c|c|c|c|}
\hline Autor & $\begin{array}{l}\text { Ano de } \\
\text { publicação }\end{array}$ & Ano de coleta & Local de coleta & Amostra & $\begin{array}{l}\text { Faixa etária/ano } \\
\text { escolar }\end{array}$ & Seleção \\
\hline Barbosa Filho et al. ${ }^{17}$ & 2015 & 2014 & Fortaleza, Ceará & 1.085 & $7^{\circ}$ ao $9^{\circ}$ ano $\mathrm{EF}$ & Aleatória \\
\hline Christofaro et al. ${ }^{18}$ & 2015 & 2011 & Londrina, Paraná & 1.231 & 14 a 17 & Conveniência \\
\hline Farah et al. ${ }^{19}$ & 2015 & 2011 & Estado de Pernambuco & 4.619 & 14 a 19 & Aleatória \\
\hline Ferrari et al..$^{20}$ & 2015 & 2012 a 2013 & São Caetano do Sul, São Paulo & 485 & 9 a 11 & Aleatória \\
\hline Ferrari et al..$^{21}$ & 2015 & 2012 a 2013 & São Caetano do Sul, São Paulo & 485 & 9 a 11 & Aleatória \\
\hline Fronza et al. ${ }^{22}$ & 2015 & 2010 & São Bonifácio, Santa Catarina & 283 & 10 a 19 & Conveniência \\
\hline Gordia et al. ${ }^{23}$ & 2015 & 2011 a 2012 & Amargosa, Bahia & 1.044 & 6 a 18 & Aleatória \\
\hline Leme, Philippi ${ }^{24}$ & 2015 & 2014 & São Paulo, São Paulo & 253 & $2^{\circ}$ e $3^{\circ}$ ano $\mathrm{EM}$ & Conveniência \\
\hline Menezes, Duarte ${ }^{25}$ & 2015 & 2011 & Escolas da rede estadual de Sergipe & 3.992 & 14 a 19 & Aleatória \\
\hline Romero et a $\mathrm{a}^{26}$ & 2015 & 2009 & Piracicaba, São Paulo & 454 & 10 a 14 & Aleatória \\
\hline Azeredo et al. ${ }^{27}$ & 2016 & 2012 & $\begin{array}{l}\text { Todas as capitais do Brasil, Distrito Federal } \\
\text { e outras cidades }\end{array}$ & 109.104 & $9^{\circ}$ ano $\mathrm{EF}$ & Aleatória \\
\hline Barbosa et al. ${ }^{28}$ & 2016 & ND & Florianópolis, Santa Catarina & 135 & 10 a 17 & Conveniência \\
\hline Felden et al. ${ }^{29}$ & 2016 & ND & Maravilha, Santa Catarina & 516 & 10 a 19 & Aleatória \\
\hline Ferreira et al. ${ }^{30}$ & 2016 & 2013 & Pelotas, Rio Grande do Sul & 8.661 & $\begin{array}{l}5^{\circ} \text { ano } \mathrm{EF} \text { ao } 3^{\circ} \text { ano } \\
\mathrm{EM}\end{array}$ & Aleatória \\
\hline Leme et al. ${ }^{31}$ & 2016 & 2014 & São Paulo, São Paulo & 253 & $2^{\circ}$ e $3^{\circ}$ ano $\mathrm{EM}$ & Aleatória \\
\hline Matsudo et al. ${ }^{32}$ & 2016 & 2012 a 2013 & São Caetano do Sul, São Paulo & 485 & 9 a 11 & Aleatória \\
\hline Moraes et al. ${ }^{33}$ & 2016 & $\begin{array}{l}\text { HELENA: } 2006 \text { a } \\
\text { 2007/BRACAH: } 2007\end{array}$ & Multicêntrico* & 1.934 & $\begin{array}{l}\text { HELENA: } 12,5-17,5 / \\
\text { BRACAH: } 14-17,5\end{array}$ & Aleatória \\
\hline Regis et al. ${ }^{34}$ & 2016 & 2011 & $\begin{array}{l}\text { Escolas da rede pública estadual EM } \\
\text { estado de Pernambuco }\end{array}$ & $6 . .234$ & 14 a 19 & Aleatória \\
\hline Silva, Silva ${ }^{35}$ & 2016 & 2011 & Aracajú, Sergipe & 739 & 14 a 18 & Conveniência \\
\hline Costa et al. ${ }^{36}$ & 2017 & 2012 & Florianópolis, Santa Catarina & 571 & 7 a 12 & Conveniência \\
\hline Ferreira et al. ${ }^{37}$ & 2017 & 2012 & $\begin{array}{l}\text { Todas as capitais do Brasil, Distrito Federal } \\
\text { e outras cidades }\end{array}$ & 109.104 & $9^{\circ}$ ano $\mathrm{EF}$ & Aleatória \\
\hline Greca, Silva ${ }^{38}$ & 2017 & 2011 & Londrina, Paraná & 455 & 8 a 17 & Conveniência \\
\hline Ribeiro et al. ${ }^{39}$ & 2017 & 2014 & Frei Paulo, Sergipe & 975 & 4 a 17 & Conveniência \\
\hline Silva et al. ${ }^{40}$ & 2017 & $2004,2008,2011$ & Pelotas, Rio Grande do Sul & 3.382 & 11,15 e 18 & Conveniência \\
\hline Tebar et al. ${ }^{41}$ & 2017 & ND & Londrina, Paraná & 1.231 & 14 a 17 & Conveniência \\
\hline Bacil et al. ${ }^{42}$ & 2018 & 2015 & Curitiba, Paraná & 117 & 9 a 15 & Aleatória \\
\hline Coledam et al. ${ }^{43}$ & 2018 & 2012 & Londrina, Paraná & 681 & 10 a 17 & Aleatória \\
\hline Costa et al. ${ }^{44}$ & 2018 & 2015 & $\begin{array}{l}\text { Todas as capitais do Brasil, Distrito Federal } \\
\text { e outras cidades }\end{array}$ & 102.072 & $9^{\circ}$ ano $\mathrm{EF}$ & Aleatória \\
\hline Leme et al. ${ }^{45}$ & 2018 & 2015 & São Paulo, São Paulo & 253 & $2^{\circ}$ e $3^{\circ}$ ano $\mathrm{EM}$ & Aleatória \\
\hline Matias et al. ${ }^{46}$ & 2018 & 2015 & $\begin{array}{l}\text { Todas as capitais do Brasil, Distrito Federal } \\
\text { e outras cidades }\end{array}$ & 102.072 & $9^{\circ}$ ano $\mathrm{EF}$ & Aleatória \\
\hline Oliveira et al. ${ }^{47}$ & 2018 & 2011 & $\begin{array}{l}\text { Escolas da rede pública estadual EM } \\
\text { estado de Pernambuco }\end{array}$ & 6.264 & 14 a 19 & Aleatória \\
\hline Oliveira et al. ${ }^{48}$ & 2018 & 2011 a 2012 & $\begin{array}{l}\text { Todas as capitais do Brasil e Distrito } \\
\text { Federal }\end{array}$ & 14.653 & $9^{\circ}$ ano $\mathrm{EF}$ & Aleatória \\
\hline Silva et al..$^{49}$ & 2018 & 2012 & $\begin{array}{l}\text { Todas as capitais do Brasil, Distrito Federal } \\
\text { e outras cidades }\end{array}$ & 109.104 & $9^{\circ}$ ano EF & Aleatória \\
\hline Santos et al. ${ }^{50}$ & 2018 & 2016 & Dourados, Mata Grosso do Sul & 274 & 12 a 18 & Aleatória \\
\hline Ulbricht et al. ${ }^{51}$ & 2018 & 2014 a 2016 & Região metropolitana de Curitiba, Paraná & 675 & 11 a 18 & Conveniência \\
\hline Barbosa Filho et al. ${ }^{52}$ & 2019 & 2014 & Fortaleza, Ceará & 1.085 & 11 a 18 & Aleatória \\
\hline Silva et al..$^{53}$ & 2019 & 2015 & Todas as capitais do Brasil & 12.220 & 11 a 19 & Aleatória \\
\hline Straatmann et al..$^{54}$ & 2019 & 2010 a 2012 & Região metropolitana do Rio de Janeiro & 810 & EF e EM & Conveniência \\
\hline
\end{tabular}

HELENA = Healthy Lifestyle in Europe by Nutrition in Adolescence; BRACAH = Brazilian Cardiovascular Adolescent Healtb; Multicêntrico* = HELENA, realizado em Atenas e Heraklion (Grécia), Dortmund (Alemanha), Ghent (Bélgica), Lille (França), Pécs (Hungria), Roma (Itália), Estocolmo (Suécia), Viena (Áustria), Zaragoza (Espanha) e BRACAH, realizado em Maringá, Paraná, Brasil; ND = Não descrito; EF = Ensino Fundamental; EM = Ensino Médio. 
Tabela 2-Características metodológicas dos estudos avaliados.

\begin{tabular}{|c|c|c|c|}
\hline Autor & Pontos de corte e indicadores & Instrumento de avaliação & Exposição ao Comportamento Sedentário \\
\hline Barbosa Filho et al. ${ }^{17}$ & Tela $2 \mathrm{~h} / \mathrm{d}$ & $\mathrm{QDE}^{* *}$ & ND \\
\hline Christofaro et al..$^{18}$ & Tela $(<2 \mathrm{~h} / \mathrm{d}, 2-3 \mathrm{~h} / \mathrm{d}, 3-4 \mathrm{~h} / \mathrm{d},>4 \mathrm{~h} / \mathrm{d})$ & QDE & $\geq 2 \mathrm{~h} / \mathrm{d}: \mathrm{M}=93,8 \% / \mathrm{F}=87,2 \%$ \\
\hline Farah et al..$^{19}$ & Tela + Sentado $\geq 3 \mathrm{~h} / \mathrm{d}$ & Questionário GSHS (versão adaptada) & Total $=50,9 \% / \mathrm{M}=38,4 \% / \mathrm{F}=61,6 \%$ \\
\hline Ferrari et al. ${ }^{20}$ & $\begin{array}{l}\mathrm{TV}>2 \mathrm{~h} / \mathrm{d}+\leq 25 \text { contagens de } \\
\text { atividade } / 15 \mathrm{~s}\end{array}$ & $\begin{array}{l}\text { ISCOLE Diet and Lifestyle } \\
\text { Questionnaire e Acelerometria }\end{array}$ & $\begin{array}{l}\text { Total semana }=34,2 \% / \mathrm{fds}=54,4 \% \\
\text { M semana }=15,6 \% / \mathrm{fds}=28,1 \% \\
\mathrm{~F} \text { semana }=18,6 \% / \mathrm{fds}=26,4 \%\end{array}$ \\
\hline Ferrari et al. ${ }^{21}$ & $\leq 25$ contagens de atividade $/ 15 \mathrm{~s}$ & Acelerometria & $\begin{array}{l}\text { Média total }=500 \mathrm{~min} / \mathrm{d} \\
\mathrm{M}=492,5 \mathrm{~min} / \mathrm{d} / \mathrm{F}=507,3 \mathrm{~min} / \mathrm{d}\end{array}$ \\
\hline Fronza et al. ${ }^{22}$ & $\mathrm{TV} \geq 2 \mathrm{~h} / \mathrm{d}$ & QDE & Total semana $=76,7 \% / \mathrm{fds}=78,4 \%$ \\
\hline Gordia et al. ${ }^{23}$ & TV $3 \mathrm{~h} / \mathrm{d}$ & Questionário PAQ-C & $\begin{array}{l}\text { Média total }=3,7 \mathrm{~h} / \mathrm{d} \\
\text { crianças }=3,8 \mathrm{~h} / \mathrm{d} / \text { adolescentes }=3,6 \mathrm{~h} / \mathrm{d}\end{array}$ \\
\hline Leme, Philippi ${ }^{24}$ & Tela $>2 \mathrm{~h} / \mathrm{d}$ & ND & $\mathrm{GE}=75,4 \% / \mathrm{GC}=79,3 \%$ \\
\hline Menezes, Duarte $e^{25}$ & Sentado $>2 \mathrm{~h} / \mathrm{d}$ & Questionário baseado no GSHS & $\begin{array}{l}\text { Total }=46,7 \% \\
\mathrm{M}=42,5 \% / \mathrm{F}=49,4 \%\end{array}$ \\
\hline Romero et al. ${ }^{26}$ & Tela h/sem & Questionário QACV & $\begin{array}{l}\text { Média total }=1.692,1 \mathrm{~min} / \mathrm{sem} \\
\mathrm{M}=1.801,5 \mathrm{~min} / \mathrm{sem} / \mathrm{F}=1.597,1 \mathrm{~min} / \mathrm{sem}\end{array}$ \\
\hline Azeredo et al. ${ }^{27}$ & Tela + Sentado $(<1 \mathrm{~h} / \mathrm{d}$ até $>8 \mathrm{~h} / \mathrm{d})$ & PeNSE 2012 & ND \\
\hline Barbosa et al. ${ }^{28}$ & Tela $(\mathrm{TV} \geq 4$ h/d; PC-VG $\geq 3$ h/d) & QDE & $\begin{array}{l}\text { TV: } \text { Total }=42,1 \% / M=41,4 \% / F=43,2 \% / \\
\text { PC-VG: } \text { Total }=38,6 \% / \mathrm{M}=50 \% / F=20,5 \%\end{array}$ \\
\hline Felden et al. ${ }^{29}$ & $\begin{array}{l}\text { Tercis de tempo sentado }(\text { pouco sentado = } \\
0 \text { a } 300 \mathrm{~min} / \mathrm{d} \text {; tempo sentado moderado = } \\
301 \text { a } 479 \mathrm{~min} / \mathrm{d} ; \\
\text { muito tempo sentado = acima } 480 \mathrm{~min} / \mathrm{d} \text { ) }\end{array}$ & Questionário IPAQ(versão longa) & $\begin{array}{l}\text { Média total }=382,2 \mathrm{~min} / \mathrm{d} / \mathrm{M}=352,1 \mathrm{~min} / \mathrm{d} \\
/ \mathrm{F}=413,6 \mathrm{~min} / \mathrm{d}\end{array}$ \\
\hline Ferreira et al. ${ }^{30}$ & Tela + Sentado $\geq 2 \mathrm{~h} / \mathrm{d}$ & $\begin{array}{l}\text { Versão adaptada do questionário } \\
\text { HELENA }\end{array}$ & Total semana $=69,2 \% / \mathrm{fds}=79,6 \%$ \\
\hline Leme et al. ${ }^{31}$ & Tela $(0 \mathrm{~h} / \mathrm{d}$ até $>5 \mathrm{~h} / \mathrm{d})$ & $\mathrm{QDE}^{* * * *}$ & $\begin{array}{l}\text { Média: } \\
\text { GE: semana = 4,24 h/d / fds = 3,82 h/d / GC: } \\
\text { semana }=3,43 \mathrm{~h} / \mathrm{d} / \mathrm{fds}=4,62 \mathrm{~h} / \mathrm{d}\end{array}$ \\
\hline Matsudo et al. ${ }^{32}$ & $\leq 25$ contagens de atividade $/ 15 \mathrm{~s}$ & Acelerometria & Média total $=499,7 \mathrm{~min} / \mathrm{d}$ \\
\hline Moraes et al. ${ }^{33}$ & Tela h/sem & $\mathrm{QDE}^{*}$ & ND \\
\hline Regis et al. ${ }^{34}$ & Tela + Sentado $\geq 4 \mathrm{~h} / \mathrm{d}$ & Questionário GSHS & $\begin{array}{l}\text { TV: } \text { Total }=13,2 \% / \text { PC-VG: } \text { Total }=9,5 \% / \\
\text { Sentado: } \text { Total }=14,6 \%\end{array}$ \\
\hline Silva; Silva ${ }^{35}$ & $\mathrm{TV}>2 \mathrm{~h} / \mathrm{d}$ & Versão brasileira do YRBSS & Total $=66 \%$ \\
\hline Costa et al..$^{36}$ & $\leq 25$ contagens de atividade $/ 15 \mathrm{~s}$ & Acelerometria & $\begin{array}{l}\text { Média: } \\
\text { Dia escolar: Total }=132,6 \min / \mathrm{M}=126,9 \\
\mathrm{~min} / \mathrm{F}=137,5 \mathrm{~min} / 7 \text { a } 9 \text { anos de idade }= \\
126,3 \mathrm{~min} / 10 \text { a } 12 \text { anos de idade }=144,2 \mathrm{~min}\end{array}$ \\
\hline Ferreira et al. ${ }^{37}$ & Tela + Sentado $\geq 3 \mathrm{~h} / \mathrm{d}$ & PeNSE 2012 & Total $=62 \% / \mathrm{M}=59,1 \% / \mathrm{F}=64,6 \%$ \\
\hline Greca; Silva ${ }^{38}$ & Tela $\geq 2 \mathrm{~h} / \mathrm{d}$ & Questionário PAQ-C & Total $=66,8 \%$ \\
\hline Ribeiro et al. ${ }^{39}$ & Tela $>2 \mathrm{~h} / \mathrm{d}$ & QDE & $\begin{array}{l}\text { Escolares sem excesso de peso: } 51,67 \% \text { / } \\
\text { Escolares com excesso de peso: } 47,06 \%\end{array}$ \\
\hline Silva et al. ${ }^{40}$ & Tela (h/dia de semana) & $\mathrm{QDE}^{* * * * *}$ & $\begin{array}{l}\text { Média: } \\
\text { Grupo } 1 \text { : Idade } 11=5,51 \mathrm{~h} / \text { Idade } 15=6,88 \\
\text { h / Idade } 18=6,38 \mathrm{~h} \\
\text { Grupo } 2: \text { Idade } 11=3,33 \mathrm{~h} / \text { Idade } 15=3,64 \\
\text { h / Idade } 18=3,46 \mathrm{~h} \\
\text { Grupo } 3: \text { Idade } 11=1,37 \mathrm{~h} / \text { Idade } 15=1,27 \\
\text { h } / \text { Idade } 18=1,16 \mathrm{~h}\end{array}$ \\
\hline Tebar et al..$^{41}$ & Tela $\geq 22 \mathrm{~h} / \mathrm{sem}$ & ND & Baixo CS: 29,3\% / Alto CS: 70,7\% \\
\hline Bacil et al. ${ }^{42}$ & $\begin{array}{l}\text { Atividades sedentárias } \mathrm{h} / \mathrm{d}+100 \text { contagens } \\
\text { de atividade/min }\end{array}$ & QASA e Acelerometria & Elevado CS: Total = 33,3\% \\
\hline Coledam et al. ${ }^{43}$ & $\begin{array}{l}\text { Tela }(<1 \mathrm{~h} / \mathrm{d}, 1 \mathrm{~h} / \mathrm{d}, 2 \mathrm{~h} / \mathrm{d}, 3 \mathrm{~h} / \mathrm{d}, 4 \mathrm{~h} / \mathrm{d}, \geq \\
5 \mathrm{~h} / \mathrm{d})\end{array}$ & QDE & ND \\
\hline Costa et al. ${ }^{44}$ & Tela + Sentado $>2 \mathrm{~h} / \mathrm{d}$ & PeNSE 2015 & Total $=68,1 \%$ \\
\hline
\end{tabular}

Continua... 
... continua

\begin{tabular}{|c|c|c|c|}
\hline Leme et al. ${ }^{45}$ & Tela $(0 \mathrm{~h} / \mathrm{d}$ até $\geq 5 \mathrm{~h} / \mathrm{d})$ & $\begin{array}{l}\text { Versão traduzida para o português do } \\
\text { New Moves Questionnaire }\end{array}$ & $\begin{array}{l}\text { Média: } \\
\text { GE: Dias de semana }=3,28 \mathrm{~h} / \mathrm{d} / \mathrm{fds}=4,59 \\
\mathrm{~h} / \mathrm{d} \\
\text { GC: Dias de semana }=2,97 \mathrm{~h} / \mathrm{d} / \mathrm{fds}=3,96 \\
\mathrm{~h} / \mathrm{d}\end{array}$ \\
\hline Matias et al. ${ }^{46}$ & Tela + Sentado $(1 \mathrm{~h} / \mathrm{d}$ até $9 \mathrm{~h} / \mathrm{d})$ & PeNSE 2015 & $\begin{array}{l}\text { Média: } \\
\text { Cluster } 1=2,59 \mathrm{~h} / \mathrm{d} / \text { Cluster } 2=3,85 \mathrm{~h} / \mathrm{d} / \\
\text { Cluster } 3=7,78 \mathrm{~h} / \mathrm{d}\end{array}$ \\
\hline Oliveira et al. ${ }^{47}$ & Tela + Sentado $(<2 \mathrm{~h} / \mathrm{d}, 2-4 \mathrm{~h} / \mathrm{d},>4 \mathrm{~h} / \mathrm{d})$ & Questionário GSHS & $\begin{array}{l}<2 \mathrm{~h} / \mathrm{d}: \text { Total }=9,9 \% / \mathrm{M}=8,8 \% / \mathrm{F}=10,7 \% \\
2-4 \mathrm{~h} / \mathrm{d}: \text { Total }=21,5 \% / \mathrm{M}=20,5 \% / \mathrm{F}= \\
22,1 \% \\
>4 \mathrm{~h} / \mathrm{d}: \text { Total }=68,6 \% / \mathrm{M}=70,6 \% / \mathrm{F}=67,2 \%\end{array}$ \\
\hline Oliveira et al. ${ }^{48}$ & Tela $(<2 \mathrm{~h} / \mathrm{d}, 2-4 \mathrm{~h} / \mathrm{d},>4 \mathrm{~h} / \mathrm{d})$ & $\mathrm{QDE}^{* * * * *}$ & $\begin{array}{l}\mathrm{TV}:<2 \mathrm{~h} / \mathrm{d}=30,7 \% / 2-4 \mathrm{~h} / \mathrm{d}=37,8 \% />4 \\
\mathrm{~h} / \mathrm{d}=31,5 \% \\
\mathrm{PC}-\text { Internet: }<2 \mathrm{~h} / \mathrm{d}=42,2 \% / 2-4 \mathrm{~h} / \mathrm{d}= \\
28,8 \% />4 \mathrm{~h} / \mathrm{d}=29 \%\end{array}$ \\
\hline Silva et al. ${ }^{49}$ & Lazer sedentário $>4 \mathrm{~h} / \mathrm{d}+\mathrm{TV}>2 \mathrm{~h} / \mathrm{d}$ & PeNSE 2012 & $\begin{array}{l}\text { Lazer sedentário = 37,7\% } \\
\mathrm{TV}=62,2 \%\end{array}$ \\
\hline Santos et al. ${ }^{50}$ & Tela $>2 \mathrm{~h} / \mathrm{d}$ & QDE & Total $=75,9 \%$ \\
\hline Ulbricht et al. ${ }^{51}$ & Tela $>2 \mathrm{~h} / \mathrm{d}$ & Questionário IPAQ (versão curta) & Total $=80,8 \% / \mathrm{M}=75,2 \% / \mathrm{F}=79,3 \%$ \\
\hline Barbosa Filho et al..$^{52}$ & Tela $<2 \mathrm{~h} / \mathrm{d}$ & Questionário baseado no YRBS & $\begin{array}{l}\mathrm{GE} \text { (pré): } \mathrm{TV}=28,7 \% / \mathrm{PC}-\mathrm{VG}=43,7 \% \\
\mathrm{GE} \text { (pós): } \mathrm{TV}=\text { aumento de } 6,4 \% / \mathrm{PC}-\mathrm{VG}= \\
\text { aumento de } 8,6 \% \\
\mathrm{GC} \text { (pré): } \mathrm{TV}=31,1 \% / \mathrm{PC}-\mathrm{VG}=46,6 \% \\
\mathrm{GC} \text { (pós): } \mathrm{TV}=\text { aumento de } 4,7 \% / \mathrm{PC}-\mathrm{VG}= \\
\text { aumento de } 3,7 \%\end{array}$ \\
\hline Silva et $\mathrm{al}^{53}$ & $\begin{array}{l}\text { TV }+ \text { Tela/Sentado }(\mathrm{TV}>2 \mathrm{~h} / \mathrm{d} ; \text { Tela/ } \\
\text { Sentado } \leq 2 \mathrm{~h} / \mathrm{d},>2 \mathrm{~h} / \mathrm{d} \text { até } \leq 4 \mathrm{~h} / \mathrm{d},>4 \\
\mathrm{~h} / \mathrm{d})\end{array}$ & PeNSE 2015 & $\begin{array}{l}\text { Total: } \\
0 \text { aulas EF/sem: } \mathrm{TV}=51,7 \% / \text { Sentado }>4 \\
\mathrm{~h} / \mathrm{d}=40,8 \% \\
1 \mathrm{a} 2 \text { aulas EF/sem: } \mathrm{TV}=51,4 \% / \text { Sentado }> \\
4 \mathrm{~h} / \mathrm{d}=38,3 \% \\
\geq 3 \text { aulas EF/sem: } \mathrm{TV}=56,7 \% / \text { Sentado }>4 \\
\mathrm{~h} / \mathrm{d}=38,5 \%\end{array}$ \\
\hline Straatmann et al. ${ }^{54}$ & Tela $\mathrm{min} / \mathrm{d}$ & $\begin{array}{l}\text { Questionário HBSC (relatório } \\
\text { internacional de 2005/2006) }\end{array}$ & $\begin{array}{l}\text { 2010: Média M: TV = } 195 \mathrm{~min} / \mathrm{d} / \mathrm{VG}-\mathrm{PC}= \\
\text { 142,1 } \mathrm{min} / \mathrm{d} \\
\quad \mathrm{F}: \mathrm{TV}=200,4 \mathrm{~min} / \mathrm{d} / \mathrm{VG}-\mathrm{PC} \\
=104,4 \mathrm{~min} / \mathrm{d} \\
\text { 2011: Média M: TV }=192,7 \mathrm{~min} / \mathrm{d} / \mathrm{VG}-\mathrm{PC} \\
=166,6 \mathrm{~min} / \mathrm{d} \\
\quad \mathrm{F}: \mathrm{TV}=193,5 \mathrm{~min} / \mathrm{d} / \mathrm{VG}-\mathrm{PC} \\
=181,6 \mathrm{~min} / \mathrm{d} \\
\text { 2012: Média M: TV }=179,2 \mathrm{~min} / \mathrm{d} / \mathrm{VG}-\mathrm{PC} \\
=168,8 \mathrm{~min} / \mathrm{d} \\
\quad \mathrm{F}: \mathrm{TV}=177,5 \mathrm{~min} / \mathrm{d} / \mathrm{VG}-\mathrm{PC} \\
=161,2 \mathrm{~min} / \mathrm{d}\end{array}$ \\
\hline
\end{tabular}

$\mathrm{s}=$ segundos $\mathrm{min}=\operatorname{minutos} ; \mathrm{h}=$ horas $\mathrm{h} / \mathrm{d}=$ horas por dia $\mathrm{h} /$ dia de semana $=$ horas por dia de semana $\mathrm{h} / \mathrm{sem}=\mathrm{horas}$ por semana; $\mathrm{min} / \mathrm{d}$ $=$ minutos por dia $; \mathrm{min} / \mathrm{sem}=$ minutos por semana; contagens de atividade $/ \mathrm{min}=$ contagens de atividade por minuto; fds = final de semana; $\mathrm{EF} /$ sem = educação física por semana $\mathrm{PC}=$ computador; $\mathrm{VG}=$ vídeo game $; \mathrm{TV}=$ televisão; $\mathrm{M}=$ masculino; $\mathrm{F}=$ feminino; $\mathrm{QACV}=$ Questionnaire for Adolescents Computerized Version; PeNSE = Pesquisa Nacional de Saúde do Escolar; QDE = Questionário desenvolvido pelo estudo; GSHS = Global School-based Student Health Survey; ISCOLE = International Study of Childhood Obesity, Lifestyle and the Environment, PAQ-C = Physical Activity Questionnaire for Older Children; YRBSS = Youth Risk Behavior Surveillance System; YRBS = Youth Risk Behavior Survey; IPAQ = International Physical Activity Questionnaire; HELENA = Healtby Lifestyle in Europe by Nutrition in Adolescence; HBSC = Health Behaviour in School-Aged Children; QASA = Questionário de Atividades Sedentárias para Adolescentes; QDE* $=$ Questionário traduzido e "harmonizado" entre países, já utilizado por Moraes et al. ${ }^{83}$, Rey-Lopez et al. ${ }^{84,85}$; QDE** = Instrumento validado/adaptado para jovens brasileiros; QDE $^{* * *}=$ Medida modificada previamente utilizada com adolescentes (meninas) por Neumark-Sztainer et al. ${ }^{86}$; QDE ${ }^{* * * *}=$ Questionário testado em 2011 com 1049 adolescentes do $9^{\circ}$ ano de escolas públicas de Brasília, Distrito Federal; QDE***** = Utilizou-se mais de um questionário; ND = Não descrito; GE = Grupo experimental; GC = Grupo controle; CS = comportamento sedentário.

maiores quantidades de doces, em comparação àqueles menos expostos ${ }^{18}$. Além desses malefícios, a alta exposição ao comportamento sedentário pode resultar no aco- metimento da síndrome metabólica. Esta se refere a um conjunto de fatores de risco que favorecem o aumento de risco de eventos cardiovasculares, doenças das artérias 
Tabela 3 - Características descritivas dos estudos avaliados.

\begin{tabular}{|c|c|c|c|}
\hline Autor & $\begin{array}{c}\text { Variável } \\
\text { (desfecho X exposição) }\end{array}$ & Desenho & Variáveis \\
\hline Barbosa Filho et al. ${ }^{17}$ & $\mathrm{D}$ & ERC & Comportamentos em saúde \\
\hline Christofaro et al. ${ }^{18}$ & $\mathrm{D}$ & TR & Sobrepeso, nutrição e atividade física \\
\hline Farah et al. ${ }^{19}$ & $\mathrm{E}$ & TR & FC em repouso \\
\hline Ferrari et al..$^{20}$ & $\mathrm{D}$ & TR & Atividade física \\
\hline Ferrari et al..$^{21}$ & $\mathrm{D}$ & TR & Composição corporal \\
\hline Fronza et al. ${ }^{22}$ & $\mathrm{D}$ & TR & Características sociodemográficas e fatores de risco à saúde \\
\hline Gordia et al. ${ }^{23}$ & $\mathrm{E}$ & TR & Hiperglicemia \\
\hline Leme, Philippi ${ }^{24}$ & $\mathrm{E}$ & $\mathrm{ERC}$ & Nutrição, obesidade e atividade física \\
\hline Menezes, Duarte ${ }^{25}$ & $\mathrm{D}$ & TR & Características sociodemográficas \\
\hline Romero et al. ${ }^{26}$ & $\mathrm{D}$ & TR & Excesso de peso corporal \\
\hline Azeredo et al. ${ }^{27}$ & $\mathrm{E}$ & TR & Comportamentos em saúde e características sociodemográficas \\
\hline Barbosa et al..$^{28}$ & $\mathrm{E}$ & TR & Sintomas depressivos \\
\hline Felden et al..$^{29}$ & $\mathrm{D}$ & TR & Sono \\
\hline Ferreira et al. ${ }^{30}$ & $\mathrm{D}$ & TR & Características sociodemográficas e nível de atividade física \\
\hline Leme et al. ${ }^{31}$ & $\mathrm{E}$ & ERC & Obesidade \\
\hline Matsudo et al. ${ }^{32}$ & $\mathrm{E}$ & TR & Indicadores de nível socioeconômico, atividade física e sobrepeso/obesidade \\
\hline Moraes et al. ${ }^{33}$ & $\mathrm{E}$ & TR & Pressão arterial \\
\hline Regis et al. ${ }^{34}$ & $\mathrm{D}$ & TR & Características sociodemográficas \\
\hline Silva, Silva ${ }^{35}$ & $\mathrm{E}$ & TR & Prática de futebol \\
\hline Costa et al. ${ }^{36}$ & $\mathrm{D}$ & TR & Características sociodemográficas, medidas antropométricas, aulas de E.F., desempenho acadêmico \\
\hline Ferreira et al. ${ }^{37}$ & $\mathrm{D}$ & TR & Excesso de peso corporal \\
\hline Greca, Silva ${ }^{38}$ & $\mathrm{D}$ & TR & Recesso escolar \\
\hline Ribeiro et al. ${ }^{39}$ & $\mathrm{E}$ & $\mathrm{CO}$ & Excesso de peso corporal \\
\hline Silva et al. ${ }^{40}$ & $\mathrm{D}$ & $\mathrm{CO}$ & Função pulmonar \\
\hline Tebar et al. ${ }^{41}$ & $\mathrm{E}$ & TR & Obesidade abdominal \\
\hline Bacil et al. ${ }^{42}$ & $\mathrm{D}$ & TR & Validação de questionário \\
\hline Coledam et al. ${ }^{43}$ & $\mathrm{E}$ & TR & Aulas de E.F. \\
\hline Costa et al. ${ }^{44}$ & $\mathrm{E}$ & $\mathrm{TR}$ & Nutrição \\
\hline Leme et al..$^{45}$ & $\mathrm{E}$ & ERC & Obesidade \\
\hline Matias et al. ${ }^{46}$ & $\mathrm{D}$ & $\mathrm{TR}$ & Características sociodemográficas \\
\hline Oliveira et al. ${ }^{47}$ & $\mathrm{E}$ & TR & Pressão arterial \\
\hline Oliveira et al..$^{48}$ & $\mathrm{E}$ & TR & Nutrição e atividade física \\
\hline Silva et al. ${ }^{49}$ & $\mathrm{E}$ & TR & Asma \\
\hline Santos et al..$^{50}$ & $\mathrm{E}$ & TR & Síndrome metabólica \\
\hline Ulbricht et al. ${ }^{51}$ & $\mathrm{E}$ & TR & Sobrepeso/obesidade \\
\hline Barbosa Filho et al..$^{52}$ & $\mathrm{D}$ & ERC & Comportamentos em saúde \\
\hline Silva et al. ${ }^{53}$ & $\mathrm{E}$ & TR & Aulas de E.F. \\
\hline Straatmann et al..$^{54}$ & $\mathrm{D}$ & $\mathrm{CO}$ & Atividade física \\
\hline
\end{tabular}

$\mathrm{E}=$ Exposição; $\mathrm{D}=$ Desfecho; $\mathrm{TR}=$ Transversal $; \mathrm{ERC}=$ Ensaio randomizado controlado $; \mathrm{CO}=$ Coorte FC = Frequência cardíaca $;$ E.F. $=$ Educação Física.

coronárias, diabetes, mortalidade por todas as causas e por doenças cardiovasculares ${ }^{3}$. Indivíduos que despendem maior tempo em comportamento sedentário aumentam em $73 \%$ o risco de desenvolver essa síndrome ${ }^{64}$.

A elevada exposição dos adolescentes ao comportamento sedentário ${ }^{65}$ também pode gerar prejuízos à sua vida escolar. Revisão sistemática que investigou a asso- ciação entre atividade física, comportamento sedentário e qualidade de vida de crianças e adolescentes ${ }^{66}$, revelou que aqueles que reportaram maior tempo gasto em atividades sedentárias apresentaram menor qualidade de vida no domínio escolar. A prevalência de sintomas depressivos aumentou com o maior tempo sedentário ${ }^{67}$, o qual também se associou à evasão escolar ${ }^{68}$. 
Quanto a estratégias adotadas para a redução do comportamento sedentário, intervenções que envolveram a participação da família, intervenção em hábitos e rotinas e que se utilizaram de tecnologia (como equipamentos que regulam o tempo despendido com TV, por exemplo) demonstraram resultados satisfatórios na redução do comportamento sedentário ${ }^{14}$. Porém, é importante destacar que grande parte dos estudos de intervenção tem focado em outros desfechos, como atividade física e dieta. Assim, tem se tratado o comportamento sedentário apenas como uma variável secundária, sendo minoria os experimentos que o investigaram como principal desfecho ${ }^{13,69}$. Em revisão sistemática e meta-análise com intervenções em comportamento sedentário e atividade física, baseadas em sala de aula, não foram encontrados estudos que investigassem somente o comportamento sedentário ${ }^{70}$. Ainda assim, mesmo apresentando pequeno efeito, esse tipo de experimento apresenta resultados mais satisfatórios que aqueles que intervêm no comportamento sedentário combinado à outras variáveis ${ }^{13,14}$. Com isso, sugere-se que para se reduzir a exposição ao comportamento sedentário seja necessário um foco maior nesse desfecho e não somente a busca pela sua redução por meio do aumento do nível de atividade física ${ }^{71}$.

Em nossa revisão foi encontrado que somente $13,1 \%$ dos estudos apresentaram desenho de pesquisa experimental. A maior parte era de corte transversal $(78,9 \%)$. Isto pode, entre outros fatores, ser justificado pela vantagem produtivista desse tipo de pesquisa, uma vez que estudos experimentais e de coorte, por exemplo, exigem maior tempo de coleta e custo mais elevado ${ }^{72}$. Por outro lado, trata-se de uma tarefa complexa (haja vista que se trata de uma mudança comportamental) na qual estão envolvidos hábitos e práticas diárias realizados de forma automática e a atratividade de equipamentos eletrônicos baseados em tela ${ }^{14,69}$. Deste modo, verifica-se a necessidade da realização de pesquisas que desenvolvam práticas voltadas para a redução do comportamento sedentário, as quais possam promover uma melhor compreensão de como alterações políticas e ambientais contribuem para a sua redução $\mathrm{O}^{71}$.

Revisão sistemática sobre Epidemiologia da Atividade Física no Brasil mostrou que 11\% das pesquisas na área foram produzidas no Nordeste ${ }^{73}$, no período de 1990 a 2005. Todavia, em nossa revisão o número de estudos advindos dessa região foi superior a $23 \%$. Esse crescimento da contribuição da região Nordeste para a área de Atividade Física e Saúde tem sido significan- te nos últimos anos. Alguns fatores podem explicá-lo, como a realização de simpósios e congressos nacionais e internacionais com discussão da temática, a criação e consolidação do primeiro Programa de Pós-Graduação em Educação Física no Nordeste e a elevação da quantidade de doutores nessa região ${ }^{74}$. Por outro lado, nossos resultados mostram que o Sul ainda é a região de maior produção da temática no Brasil.

É possível avaliar o comportamento sedentário de diversas formas, como pelo tempo gasto com TV, computador, com leitura e ouvindo música deitado ou sentado, por exemplo9. Para essa mensuração, existe também uma variedade de instrumentos, tanto objetivos (acelerômetros, inclinômetros, observação direta, entre outros) quanto subjetivos (questionários de autorrelato, diários e recordatórios) ${ }^{75}$. Na presente revisão foi baixo o número de trabalhos que fizeram uso de medidas diretas para mensurar o comportamento sedentário (13,1\%), sendo escolhida a acelerometria. $\mathrm{O}$ acelerômetro é o meio mais utilizado em pesquisas que se utilizam de métodos objetivos ${ }^{9}$, porém o mesmo deve ser usado com precaução devido à inexistência de protocolos padronizados para identificação do comportamento sedentário ${ }^{75}$ e por sua limitação de não identificar o tipo de atividade que é realizada no tempo sedentário ${ }^{76}$. Tais fatores podem favorecer a utilização do autorrelato ${ }^{9}$. Ainda assim, a inserção de medidas objetivas por estudos futuros pode contribuir de forma importante para a pesquisa em comportamento sedentário, haja vista que tais instrumentos tendem a complementar os dados obtidos por meio do autorrelato e a diminuir o viés de memória ${ }^{55}$. Torna-se importante, portanto, a realização de estudos a fim de elucidar os questionamentos sobre o uso de tais instrumentos ${ }^{77}$, considerando também que ainda não há como afirmar se os métodos diretos são melhores que os indiretos e vice-versa, uma vez que ambos apresentam vantagens e desvantagens ${ }^{78}$. Assim, deve-se atentar ao método mais adequado para alcançar os objetivos propostos.

Tempo de tela foi o indicador mais utilizado para caracterizar o comportamento sedentário nos estudos analisados. $\mathrm{O}$ ponto de corte de maior prevalência foi o de duas horas por dia, assim como encontrado em outras revisões sobre comportamento sedentário em adolescentes brasileiros ${ }^{55,59}$. Nota-se, entretanto, certa heterogeneidade entre as pesquisas exploradas (tempo de tela $>2$ horas/dia; tela $\geq 22$ horas/semana; tela + tempo sentado $\geq 4$ horas/dia; tercis de tempo sentado) e uma ampla variedade de pontos de corte $(\mathrm{n}=17)$. Ainda 
que a maioria dos trabalhos tenha considerado o ponto de corte prescrito e recomendado por importantes órgãos e pesquisadores ${ }^{79-81}-2$ horas diárias - essa falta de padronização pode ocasionar na publicação de diferentes recomendações acerca do tempo de exposição ao comportamento sedentário ${ }^{59}$. Isto pode, inclusive, gerar dúvida entre os pesquisadores da área e principalmente entre a população em geral.

Diferentemente de outras revisões que incluíram apenas pesquisas observacionais ${ }^{55,59}$ e não reportaram o ponto de corte utilizado para se estimar o comportamento sedentário ${ }^{82}$, o presente estudo apresenta os pontos de corte dos trabalhos selecionados e não excluiu aqueles que não o fizeram. Além disso, selecionou estudos de desenho experimental. Tais fatores colocam-se como pontos fortes desta revisão, haja vista a necessidade de se discutir qual o ponto de corte a ser utilizado e contribuir na sumarização de dados para futuras discussões. Soma-se a isso o decréscimo que se deu no Brasil referente à produção de pesquisas em comportamento sedentário entre os anos de 2015 e 2017 (como observado em nossos resultados), o que torna fundamental o entendimento de como tem sido estudado o comportamento sedentário em adolescentes brasileiros, reforçando a importância do presente estudo. Algumas das limitações desta revisão sistemática foram a limitação temporal e de base de dados necessárias para a viabilidade do estudo, bem como a ausência de avaliação da qualidade dos manuscritos.

Diante do exposto, nossos achados demonstraram não haver uma padronização referente aos indicadores e pontos de corte utilizados para se estimar o comportamento sedentário em adolescentes brasileiros. Isto dificulta a comparação de resultados entre as próprias pesquisas e mostra a necessidade de um consenso sobre a questão. Houve carência de trabalhos que se valem de medidas objetivas e que devem ser estimulados a fazer uso das mesmas, pois complementam os dados do autorrelato e fornecem informações mais fidedignas quanto a exposição do adolescente ao comportamento sedentário, o que pode auxiliar na elaboração de recomendações mais precisas. Notou-se também uma baixíssima prevalência de estudos experimentais, sendo necessária maior investigação quanto a eficácia de intervenções voltadas para a redução do comportamento sedentário, haja vista que seus malefícios estão bem documentados na literatura. A participação dos professores na conscientização dos adolescentes quanto aos efeitos deletérios do comportamento sedentário, pode ter importante papel na busca pela redução da exposição a esse comportamento, visto que o adolescente passa grande parte de seu dia no ambiente escolar.

\section{Conflito de interesse}

Os autores declaram não haver conflito de interesses.

\section{Financiamento}

O presente trabalho foi realizado com apoio da Coordenação de Aperfeiçoamento de Pessoal de Nível Superior - Brasil (CAPES) - Código de Financiamento 001.

\section{Contribuição dos autores}

Silva Filho RCS, participou da concepção inicial do estudo, realização da busca, redação e revisão crítica do texto. Lemes TMMA, participou da busca e da redação do texto. Sasaki JE e Gordia AP, participaram da revisão crítica do texto. Andaki ACR, participou da concepção inicial do estudo, redação e revisão crítica do texto.

\section{Agradecimentos}

Os autores agradecem ao $\mathrm{CNPq} / \mathrm{Capes}$ pela concessão da bolsa de mestrado ao primeiro autor (2017-2019) e à Pró Reitoria de Extensão da Universidade Federal do Triângulo Mineiro/UFTM pela concessão da bolsa de iniciação científica ao segundo autor.

\section{Referências}

1. Alberga AS, Sigal RJ, Goldfield G, Prud' homme D, Kenny GP. Overweight and obese teenagers: why is adolescence a critical period?: the critical period of adolescence. Pediatr Obes. 2012;7(4):261-73.

2. Owen N, Healy GN, Matthews CE, Dunstan DW. Too much sitting: the population-health science of sedentary behavior. Exerc Sport Sci Rev. 2010;38(3):105-13.

3. Sardinha LB, Magalhães J. Comportamento sedentário: epidemiologia e relevância. Rev Factores Risco. 2012;27:54-64.

4. Charansonney OL. Physical activity and aging: a life- long story. Discov Med. 2011;12(64):177-85.

5. Charansonney OL, Després J-P. Disease prevention-should we target obesity or sedentary lifestyle? Nat Rev Cardiol. 2010;7(8):468-72.

6. Busch V, Ananda Manders L, Rob Josephus de Leeuw J. Screen time associated with health behaviors and outcomes in adolescents. Am J Health Behav. 2013;37(6):819-30.

7. Instituto Brasileiro de Geografia e Estatística. Pesquisa nacional de saúde do escolar. Rio de Janeiro: Instituto Brasileiro de Geografia e Estatística; 2016.

8. Van der Ploeg HP, Hillsdon M. Is sedentary behaviour just physical inactivity by another name? Int J Behav Nutr Phys Act. 2017;14(1):142.

9. Guerra PH, Mielke GI, Garcia LMT. Comportamento sedentário. Rev Corpoconsciência. 2015;18(1):23-36.

10. Tremblay MS, Aubert S, Barnes JD, Saunders TJ, Carson V, Latimer-Cheung AE, et al. Sedentary Behavior Research Network (SBRN) - terminology consensus project process and outcome. Int J Behav Nutr Phys Act. 2017;14(1):75. 
11. Pearson N, Biddle SJH. Sedentary behavior and dietary intake in children, adolescents, and adults: a systematic review. Am J Prev Med. 2011;41(2):178-88.

12. Friedrich RR, Polet JP, Schuch I, Wagner MB. Effect of intervention programs in schools to reduce screen time: a meta-analysis. J Pediatr. 2014;90(3):232-41.

13. Ramsey BL, Rooks-Peck CR, Finnie RKC, Wethington HR, Jacob V, Fulton JE, et al. Reducing recreational sedentary screen time. Am J Prev Med. 2016;50(3):402-15.

14. Biddle SJH, Petrolini I, Pearson N. Interventions designed to reduce sedentary behaviours in young people: a review of reviews. Br J Sports Med. 2014;48(3):182-6.

15. Principais itens para relatar revisões sistemáticas e metaanálises: a recomendação PRISMA. Epidemiol Serv Saúde. 2015;24(2):335-42.

16. World Health Organization, Group on Young People and "Health for All by the Year 2000". Young people's health: a challenge for society. Geneva: World Health Organization; 1986. (World Health Organization Technical Report Series; 731).

17. Barbosa Filho VC, Lopes AS, Lima AB, Souza EA, Gubert FA, Silva KS, et al. Rationale and methods of a clusterrandomized controlled trial to promote active and healthy lifestyles among Brazilian students: the "Fortaleça sua Saúde" program. BMC Public Health. 2015;15:1212.

18. Christofaro DGD, Andrade SM, Mesas AE, Fernandes RA, Farias Júnior JC. Higher screen time is associated with overweight, poor dietary habits and physical inactivity in Brazilian adolescents, mainly among girls. Eur J Sport Sci. 2015;16(4):498-506.

19. Farah $B Q_{2}$ Christofaro DGD, Balagopal PB, Cavalcante BR, de Barros MVG, Ritti-Dias RM. Association between resting heart rate and cardiovascular risk factors in adolescents. Eur J Pediatr. 2015;174(12):1621-8.

20. Ferrari GL, Araujo TL, Oliveira L, Matsudo V, Mire E, Barreira TV, et al. Association between television viewing and physical activity in 10-Year-old Brazilian children. J Phys Act Health. 2015;12(10):1401-8.

21. Ferrari GLM, Oliveira LC, Araujo TL, Matsudo V, Barreira TV, Tudor-Locke C, et al. Moderate-to-vigorous physical activity and sedentary behavior: independent associations with body composition variables in Brazilian children. Pediatr Exerc Sci. 2015;27(3):380-9.

22. Fronza FCAO, Minatto G, Martins CR, Petroski EL. Excessive TV viewing time and ssociated factors in Brazilian adolescents from a rural area. Hum Mov Sci. 2015;16(1):20-7.

23. Gordia AP, Quadros TMB, Silva LR, Mota J. Cut-off values for step count and TV viewing time as discriminators of hyperglycaemia in Brazilian children and adolescents. Ann Hum Biol. 2015;43(5):423-9.

24. Leme ACB, Philippi ST.The "Healthy Habits, Healthy Girls" randomized controlled trial for girls: study design, protocol, and baseline results. Cad Saúde Pública. 2015;31(7):1381-94.

25. Menezes AS, Duarte MFS. Condições de vida, inatividade física e conduta sedentária de jovens nas áreas urbana e rural. Rev Bras Med Esporte. 2015;21(5):338-44.

26. Romero A, Borges C, Slater B. Patterns of physical activity and sedentary behavior associated with overweight in Brazilian adolescents. Rev Bras Ativ Fís Saúde. 2015; 20:26-35.

27. Azeredo CM, Levy RB, Peres MFT, Menezes PR, Araya R. Patterns of health-related behaviours among adolescents: a cross-sectional study based on the National Survey of School Health Brazil 2012. BMJ Open. 2016;6(11):e011571.
28. Barbosa DG, Andrade RD, Teixeira CS, Gomes Filho Neto M, Felden ÉPG. Sintomas depressivos em adolescentes em situação de vulnerabilidade social. Cad Saúde Colet. 2016;24(2):221-7.

29. Felden ÉPG, Filipin D, Barbosa DG, Andrade RD, Meyer C, Beltrame TS, et al. Adolescentes com sonolência diurna excessiva passam mais tempo em comportamento sedentário. Rev Bras Med Esporte. 2016;22(3):186-90.

30. Ferreira RW, Rombaldi AJ, Ricardo LIC, Hallal PC, Azevedo MR, Ferreira RW, et al. Prevalence of sedentary behavior and its correlates among primary and secondary school students. Rev Paul Pediatr. 2016;34(1):56-63.

31. Leme ACB, Lubans DR, Guerra PH, Dewar D, Toassa EC, Philippi ST. Preventing obesity among brazilian adolescent girls: six-month outcomes of the healthy habits, healthy girls-Brazil school-based randomized controlled trial. Prev Med. 2016;86:77-83.

32. Matsudo VKR, Ferrari GLM, Araújo TL, Oliveira LC, Mire E, Barreira TV, et al. Indicadores de nível socioeconômico, atividade física e sobrepeso/obesidade em crianças brasileiras. Rev Paul Pediatr. 2016;34(2):162-70.

33. Moraes ACF, Fernández-Alvira JM, Rendo-Urteaga T, Julián-Almárcegui C, Beghin L, Kafatos A, et al. Effects of clustering of multiple lifestyle-related behaviors on blood pressure in adolescents from two observational studies. Prev Med. 2016; 82:111-7.

34. Regis MF, Oliveira LMFT, Santos ARM, Leonidio ACR, Diniz PRB, Freitas CMSM de, et al. Urban versus rural lifestyle in adolescents: associations between environment, physical activity levels and sedentary behavior. Einstein (Sao Paulo). 2016;14(4):461-7.

35. Silva DAS, Silva RJS. Soccer practice is associated with health-related behaviours among Brazilian male adolescents. Sport Sci Health. 2016;12(2):167-74.

36. Costa BGG, Silva KS, George AM, Assis MAA. Sedentary behavior during school-time: sociodemographic, weight status, physical education class, and school performance correlates in Brazilian schoolchildren. J Sci Med Sport. 2017;20(1):70-4.

37. Ferreira NL, Claro RM, Mingoti SA, Lopes ACS. Coexistence of risk behaviors for being overweight among Brazilian adolescents. Prev Med. 2017;100:135-42.

38. Greca JPA, Silva DAS. Sedentary behavior during school recess in Southern Brazil. Percept Mot Skills. 2017;124(1):105-17.

39. Ribeiro BVDS, Mendonça RG, Oliveira LL, Lima GS, Martins-Filho PRS, Moura NPR, et al. Anthropometry and lifestyle of children and adolescent in inland of Northeastern Brazil. Rev Bras Crescimento Desenvolv Hum. 2017;27(2):140-7.

40. Silva BGC, Menezes AMB, Wehrmeister FC, Barros FC, Pratt M. Screen-based sedentary behavior during adolescence and pulmonary function in a birth cohort. Int J Behav Nutr Phys Act. 2017;14(1):82.

41. Tebar WR, Vanderlei LCM, Scarabotollo CC, Zanuto EF, Saraiva BTC, Tebar FCSG, et al. Abdominal obesity: prevalence, sociodemographic- and lifestyle-associated factors in adolescents. Rev Bras Crescimento Desenvolv Hum. 2017;27(1):56-63.

42. Bacil EDA, Watanabe PI, Silva MP, Fantinelli ER, Bozza R, Campos W. Validade de um questionário de comportamento sedentário em escolares de 9 a 15 anos de idade. Rev Bras Ciênc Saúde. 2018;22(4):341-8. 
43. Coledam DHC, Ferraiol PF, Greca JPA, Teixeira M, Oliveira AR. Aulas de educação física e desfechos relacionados à saúde em estudantes brasileiros. Rev Paul Pediatr. 2018;36(2):192-8.

44. Costa CS, Flores TR, Wendt A, Neves RG, Assunção $\mathrm{MCF}$, Santos IS. Comportamento sedentário e consumo de alimentos ultraprocessados entre adolescentes brasileiros: Pesquisa Nacional de Saúde do Escolar (PeNSE), 2015. Cad Saúde Pública. 2018;34(3):e00021017.

45. Leme ACB, Baranowski T, Thompson D, Nicklas T, Philippi ST. Sustained impact of the "Healthy Habits, Healthy Girls - Brazil" school-based randomized controlled trial for adolescents living in low-income communities. Prev Med Rep. 2018;10:346-52.

46. Matias TS, Silva KS, Silva JA, Mello GT, Salmon J. Clustering of diet, physical activity and sedentary behavior among Brazilian adolescents in the national school - based health survey (PeNSE 2015). BMC Public Health. 2018;18:1283.

47. Oliveira LMFT, Ritti-Dias RM, Farah BQ, Christofaro DGD, Barros MVG, Diniz PRB, et al. Does the type of sedentary behaviors influence blood pressurein adolescents boys and girls? A cross-sectional study. Ciênc Saúde Colet. 2018;23(8):2575-85.

48. Oliveira SS, Bauermann GM, Alves MR, Toral N. Association of dietary intake with eating behavior, screen time, and physical activity among Brazilian adolescents. Rev Chil Nutr. 2018;45(4):349-55.

49. Silva RCR, Malta D, Rodrigues L, Ramos D, Fiaccone R, Machado D, et al. Social, environmental and behavioral determinants of asthma symptoms in Brazilian middle school students - A National School Health Survey (PeNSE 2012). Int J Environ Res Public Health. 2018;15(12):2904.

50. Santos MC, Castro Coutinho APC, Souza Dantas M, Yabunaka LAM, Guedes DP, Oesterreich SA. Correlates of metabolic syndrome among young Brazilian adolescents population. Nutr J. Dec. 2018;17(1):66.

51. Ulbricht L, Campos MF, Esmanhoto E, Ripka WL. Prevalence of excessive body fat among adolescents of a south Brazilian metropolitan region and state capital, associated risk factors, and consequences. BMC Public Health. 2018;18(1):312.

52. Barbosa Filho V, Bandeira A, Minatto G, Linard J, Silva J, Costa R, et al. Effect of a multicomponent intervention on lifestyle factors among Brazilian adolescents from low human development index areas: a cluster-randomized controlled trial. Int J Environ Res Public Health. 2019;16(2):267.

53. Silva DAS, Chaput J-P, Tremblay MS. Participation frequency in physical education classes and physical activity and sitting time in Brazilian adolescents. PLoS One. 2019; 14(3):e0213785.

54. Straatmann VS, Almquist YB, Oliveira AJ, Veiga GV, Rostila M, Lopes CS. Stability and bidirectional relationship between physical activity and sedentary behaviours in Brazilian adolescents: longitudinal findings from a school cohort study. PLoS One. 2019; 14(1):e0211470.

55. Guerra PH, Farias Júnior JC, Florindo AA. Sedentary behavior in Brazilian children and adolescents: a systematic review. Rev Saúde Publica. 2016;50:9.

56. Barbosa Filho VC, Campos W, Lopes AS. Epidemiology of physical inactivity, sedentary behaviors, and unhealthy eating habits among Brazilian adolescents. Ciên Saúde Colet. 2014;19(1):173-94.

57. Silva KS, Nahas MV, Hoefelmann LP, Lopes AS, Oliveira ES. Associações entre atividade física, índice de massa corporal e comportamentos sedentários em adolescentes. Rev Bras Epidemiol. 2008;11(1):159-68.
58. Silva KS, Nahas MV, Peres KG, Lopes AS. Fatores associados à atividade física, comportamento sedentário e participação na educação física em estudantes do ensino médio em Santa Catarina, Brasil. Cad Saúde Pública. 2009;25:2187-200.

59. Oliveira A, Germano-Soares AH, Silva B, Tassitano R. Prevalência do tempo de tela como indicador do comportamento sedentário em adolescentes brasileiros: uma revisão sistemática. Motricidade. 2016;12(S2):155-64.

60. Taveras EM, Field AE, Berkey CS, Rifas-Shiman SL, Frazier AL, Colditz GA, et al. Longitudinal relationship between television viewing and leisure-time physical activity during adolescence. Pediatrics. 2007;119(2):e314-19.

61. Bergmann, GG, Tassitano RM, Bergmann MLA, Tenório MCM, Mota J. Screen time, physical activity and cardiovascular risk factors in adolescents. Rev Bras Ativ Fís Saúde. 2018;23:e0008.

62. Busch V, Ananda Manders L, Rob Josephus de Leeuw J. Screen time associated with health behaviors and outcomes in adolescents. Am J Health Behav. 2013;37(6):819-30.

63. Hale L, Guan S. Screen time and sleep among school-aged children and adolescents: a systematic literature review. Sleep Med Rev. 2015;21:50-8.

64. Edwardson CL, Gorely T, Davies MJ, Gray LJ, Khunti K, Wilmot EG, et al. Association of sedentary behaviour with metabolic syndrome: a meta-analysis. PLoS One. 2012; 7(4):e34916.

65. Wang W-Y, Hsieh Y-L, Hsueh M-C, Liu Y, Liao Y. Accelerometer-measured physical activity and sedentary behavior patterns in taiwanese adolescents. Int J Environ Res Public Health. 2019;16(22).

66. Wu XY, Han LH, Zhang JH, Luo S, Hu JW, Sun K. The influence of physical activity, sedentary behavior on healthrelated quality of life among the general population of children and adolescents: a systematic review. PLoS One. 2017;12(11).

67. Vancampfort D, Stubbs B, Firth J, Van Damme T, Koyanagi A. Sedentary behavior and depressive symptoms among 67,077 adolescents aged 12-15 years from 30 low- and middleincome countries. Int J Behav Nutr Phys Act. 2018;15.

68. Butterworth P, Leach LS. Early onset of distress disorders and high-school dropout: prospective evidence from a national cohort of australian adolescents. Am J Epidemiol. 2018;187(6):1192-8.

69. Biddle SJ, O'Connell S, Braithwaite RE. Sedentary behaviour interventions in young people: a meta-analysis. Br J Sports Med. 2011;45(11):937-42.

70. McMichan L, Gibson A-M, Rowe DA. Classroom-based physical activity and sedentary behavior interventions in adolescents: a systematic review and meta-analysis. J Phys Act Health. 2018;15(5):383-93.

71. Young DR, Hivert M-F, Alhassan S, Camhi SM, Ferguson JF, Katzmarzyk PT, et al. Sedentary behavior and cardiovascular morbidity and mortality: a science advisory from the American Heart Association. Circulation. 2016;134(13):e262-79.

72. Ramires V, Becker L, Sadovsky A, Zago A, Bielemann R, Guerra P. Evolução da pesquisa epidemiológica em atividade física e comportamento sedentário no Brasil: atualização de uma revisão sistemática. Rev Bras Ativ Fís Saúde. 2014;19(5):529-47.

73. Hallal PC, Dumith SC, Bastos JP, Reichert FF, Siqueira FV, Azevedo MR. Evolution of the epidemiological research on physical activity in Brazil: a systematic review. Rev Saúde Pública. 2007;41(3):453-60. 
74. Farias Júnior J. Atividade física e saúde no nordeste brasileiro. Rev Bras Ativ Fís Saúde. 2014;19(3):271-74.

75. Hardy LL, Hills AP, Timperio A, Cliff D, Lubans D, Morgan PJ, et al. A hitchhiker's guide to assessing sedentary behaviour among young people: deciding what method to use. J Sci Med Sport. 2013;16(1):28-35.

76. Atkin AJ, Gorely T, Clemes SA, Yates T, Edwardson C, Brage $\mathrm{S}$, et al. Methods of measurement in epidemiology: sedentary behaviour. Int J Epidemiol. 2012;41(5):1460-71.

77. Healy GN, Clark BK, Winkler EAH, Gardiner PA, Brown WJ, Matthews CE. Measurement of adults' sedentary time in population-based studies. Am J Prev Med.2011;41(2):216-27.

78. Lubans DR, Hesketh K, Cliff DP, Barnett LM, Salmon J, Dollman J, et al. A systematic review of the validity and reliability of sedentary behaviour measures used with children and adolescents: measurement of sedentary behaviour in youth. Obes Rev. 2011; 12(10):781-99.

79. Tremblay MS, Leblanc AG, Janssen I, Kho ME, Hicks A, Murumets $\mathrm{K}$, et al. Canadian sedentary behaviour guidelines for children and youth. Appl Physiol Nutr Metab. 2011;36(1):59-64.

80. Department of Health (Australia). Australia's Physical Activity and Sedentary Behaviour Guidelines and the Australian 24-Hour Movement Guidelines. Canberra: Australian Government Department of Health; 2019.
81. Amerian Academy of Pediatrics. Children, adolescents, and the media. Pediatrics. 2013;132(5):958-61.

82. Tremblay MS, LeBlanc AG, Kho ME, Saunders TJ, Larouche $\mathrm{R}$, Colley RC, et al. Systematic review of sedentary behaviour and health indicators in school-aged children and youth. Int J Behav Nutr Phys Act. 2011;8(1):98.

83. 83. Moraes ACF, Adami F, Falcão MC. Understanding the correlates of adolescents' dietary intake patterns. A multivariate analysis. Appetite. 2012;58(3):1057-62.

84. Rey-López JP, Tomas C, Vicente-Rodriguez G, GraciaMarco L, Jiménez-Pavón D, Pérez-Llamas F, et al. Sedentary behaviours and socio-economic status in Spanish adolescents: the AVENA study. Eur J Public Health. 2011;21(2):151-7.

85. Rey-López JP, Ruiz JR, Vicente-Rodríguez G, Gracia-Marco L, Manios Y, Sjöström M, et al. Physical activity does not attenuate the obesity risk of TV viewing in youth. Pediatric Obesity. 2012;7(3):240-50.

86. Neumark-Sztainer DR, Friend SE, Flattum CF, Hannan PJ, Story MT, Bauer KW, et al. New Moves-Preventing Weight-Related Problems in Adolescent Girls: A GroupRandomized Study. American Journal of Preventive Medicine. 2010;39(5):421-32.

Recebido: 03/07/2020

Aprovado: 30/09/2020

\section{Como citar este artigo:}

Silva Filho RCS, Lemes TMMA, Sasaki JE, Gordia AP, Andaki ACR. Comportamento sedentário em adolescentes brasileiros: uma revisão sistemática. Rev Bras Ativ Fís Saúde. 2020;25:e0139. DOI: 10.12820/rbafs.25e0139 